\title{
Labyrinthe
}

13 | 2002

Numéro 13

Les bourreaux

\section{Entre asservissement sexuel et despotisme politique. La figure du bourreau chez Sade}

\section{Stéphanie Genand}

\section{OpenEdition}

\section{Journals}

Édition électronique

URL : http://journals.openedition.org/labyrinthe/1510

DOI : $10.4000 /$ labyrinthe. 1510

ISSN : 1950-6031

Éditeur

Hermann

Édition imprimée

Date de publication : 15 novembre 2002

Pagination : 33-49

\section{Référence électronique}

Stéphanie Genand, «Entre asservissement sexuel et despotisme politique. La figure du bourreau chez Sade », Labyrinthe [En ligne], 13 | 2002, mis en ligne le 19 mars 2007, consulté le 30 avril 2019. URL http://journals.openedition.org/labyrinthe/1510 ; DOI : 10.4000/labyrinthe.1510

Ce document a été généré automatiquement le 30 avril 2019.

Propriété intellectuelle 


\title{
Les bourreaux
}

\section{Entre asservissement sexuel et despotisme politique. La figure du bourreau chez Sade*}

\author{
Stéphanie Genand
}

1 La figure du bourreau se trouve chez Sade au croisement de deux discours : l'un, sexuel, qui explicite la nécessité naturelle d'une soumission des êtres les plus faibles, et l'autre, politique, qui inscrit cette représentation du plaisir dans une société fondée sur le despotisme. Cette ambivalence, qui place le bourreau dans le champ de la sexualité et dans celui de l'idéologie, tient déjà à l'histoire du mot. La définition du Dictionnaire historique de la langue française d'Alain Rey signale en effet que le bourreau, avant d'être le détenteur d'un office, sous l'Ancien Régime, chargé des exécutions publiques ${ }^{1}$, est celui qui fait souffrir :

BOURREAU : n. m. (1319-1340), bourrel (v. 1355) puis bourreau (1550) est dérivé du verbe bourrer, pris au sens ancien de «maltraiter, tourmenter». [...] Le développement sémantique s'explique par le fait que le premier rôle du bourreau était de frapper et de torturer, comme l'attestent les emplois figurés. L'extinction du sens de bourrer "maltraiter" a abouti à la démotivation de bourreau, malgré bourrade et certains emplois du verbe (bourrer les côtes, etc.) ${ }^{2}$.

Il y aurait donc un lien étymologique entre la volonté d'infliger une douleur à l'autre, et la fonction politique qui incombe ensuite à celui chargé d'exécuter la condamnation d'une victime. Les deux romans de Sade que nous avons choisis dans le cadre de cette étude, Justine ou les malheurs de la vertu ${ }^{3}$ et La Philosophie dans le boudoir ${ }^{4}$, offrent une illustration frappante de cette interaction des deux domaines : le parcours de Justine, caractérisé par Sade comme une succession constante de vertus déployées par la jeune femme et de châtiments, la confronte en effet à deux séries de bourreaux. Ceux de la justice officielle, qui la condamnent pour des fautes qu'on lui impute injustement, et ceux de l'univers sans principes des libertins qu'elle côtoie ; à chaque fois, le résultat est le même puisqu'elle se voit infliger des sévices de plus en plus graves. Mais ceux-ci relèvent de deux domaines 
distincts, que Sade finit perversement par faire se rejoindre, la politique et la cruauté sexuelle. Dans La Philosophie dans le boudoir, il retrace le parcours éducatif de la jeune Eugénie, qui découvre avec ses «instituteurs immoraux » dont parle le titre complet le libertinage sous toutes ses formes, à la fois sexuelles et idéologiques, la véritable ouverture du roman étant constituée, à l'issue du cinquième dialogue, par le pamphlet intitulé « Français, encore un effort si vous voulez être républicains » : les bourreaux y sont alors des deux côtés du boudoir, à l'intérieur avec les sévices qu'ils imposent à leurs victimes, à l'extérieur dans la contre-utopie despotique imaginée par Sade.

Justine

3 Justine ou les Malheurs de la vertu, publié en 1791 alors que Sade est enfermé à la Bastille, opère un renversement de la fonction traditionnelle des bourreaux : ceux-ci désignent moins les fonctionnaires officiels que rencontre la jeune femme lors de ses démêlés avec la justice, que les libertins qu'elle croise lors de son parcours. Ce roman confère ainsi à cette figure une dimension surtout érotique: les "bourreaux", dans ce texte, seront davantage les scélérats que les représentants de la justice officielle.

4 Cette relégation des bourreaux dans le domaine du plaisir s'explique par ce que l'on désignera à partir de 1834 comme étant le "sadism", autrement dit l'association de la jouissance et de la souffrance infligée à l'autre. Les libertins de Sade ne trouvent en effet le plaisir que dans le spectacle des supplices de leurs objets sexuels, en l'occurrence Justine, ce que le personnage de Dolmancé, dans La Philosophie dans le boudoir, justifie par une théorie matérialiste fondée sur une omnipotence de la nature en chacun de nous :

« Nous voulons être émus, disent-ils, c'est le but de tout homme qui se livre à la volupté, et nous voulons l'être par les moyens les plus actifs; en partant de ce point, il ne s'agit pas de savoir si nos procédés plairont ou déplairont à l'objet qui nous sert, il s'agit seulement d'ébranler la masse de nos nerfs par le choc le plus violent possible; or, il n'est pas douteux que la douleur affectant bien plus vivement que le plaisir, les chocs résultatifs sur nous de cette sensation, produite sur les autres, seront essentiellement d'une vibration plus vigoureuse, retentiront plus énergiquement dans nous, mettront dans une circulation plus violente les esprits animaux qui, se déterminant sur les basses régions par le mouvement de rétrogradation qui leur est essentiel alors, embraseront aussitôt les organes de la volupté, et les disposeront au plaisir ${ }^{5}$. "

Si la douleur de l'autre est donc seule en mesure de provoquer l'excitation nécessaire à la jouissance, le libertin ne pourra apparaître autrement que comme un bourreau. Dans Justine, cette confusion des valeurs s'observe dans l'utilisation du mot « bourreau » ou de son synonyme " persécuteur » à propos des hommes croisés par la jeune femme, et qui lui imposent à chaque fois des supplices nécessaires à leur plaisir, qu'il s'agisse d'infibulation ou d'introduction de corps étrangers :

« On fixe mes quatre membres à terre dans le plus grand écart possible ; le bourreau qui va rétrécir les voies s'arme d'une longue aiguille au bout de laquelle est un fil ciré6" ;

«il n'est point d'expressions dans le monde qui puissent peindre ce que j'éprouve ; cependant mon bourreau jouit, sa bouche imprimée sur la mienne, semble respirer ma douleur pour en accroître ses plaisirs ${ }^{7} »$;

«le délire s'empare enfin de mon persécuteur ${ }^{8}$ ».

6 Ce ne sont que quelques exemples, mais l'utilisation de la base de données Frantext, éditée par l'Institut national de la langue française, permet de vérifier qu'à chaque fois que le mot «bourreau » apparaît dans le roman, il désigne un tortionnaire sexuel et non 
un exécuteur public; ce qui s'explique, notamment, par une conception particulière du plaisir, indissociable de la souffrance de l'autre.

7 Cet acharnement systématique des libertins sur les victimes se prolonge dans Justine ou les Malheurs de la vertu par une structure énonciative particulière : le narrateur se transforme lui aussi en bourreau à l'égard de son personnage, victime d'une persécution symbolique qui vise à lui ôter de plus en plus les moyens d'exister et de s'exprimer. Lorsqu'il compose en 1799 La Nouvelle Justine, qui développe et accentue les principaux épisodes du texte initial - lui-même seconde version du conte inédit du vivant de Sade, Les Infortunes de la vertu ${ }^{9}-$, il commence par retirer la parole à son héroïne : la narration passe en effet de la première à la troisième personne du singulier, ce qui constitue une première amputation, et ouvre la porte aux persécutions aggravées contre le personnage. Privée de la possibilité de se défendre, de justifier sa confiance dans la Providence ou de donner sa version des malheurs qui la frappent, Justine devient en quelque sorte la victime de l'auteur, libre de la plonger dans une succession ininterrompue de sévices.

Ce silence qu'on lui impose s'accompagne en outre d'une prise de distance de plus en plus marquée de Sade, qui multiplie les désignations ironiques à l'égard de son personnage : Justine devient ainsi "notre petite niaise ${ }^{10}$ ", "l'intéressante créature ${ }^{11}$ ", sans oublier que son corps, doté de facultés magiques de régénération, offre toujours la même pureté et la même fragilité aux bourreaux rencontrés. Ce phénomène surprenant, qui s'explique par le projet d'une narration elle aussi «sadique » puisqu'elle fait tout pour accentuer la souffrance du personnage, s'observe déjà dans Justine : dès qu'elle vient de subir un sévice physique susceptible de laisser des traces qui pourraient dissuader ses persécuteurs à venir, la jeune femme se remet très rapidement, et recouvre en quelques heures cette perfection qui excita ses premiers bourreaux :

"Un peu de repos succéda à ces cruelles orgies; on me laissa respirer quelques instants ; j'étais moulue, mais ce qui me surprit, ils guérirent mes plaies en moins de temps qu'ils n'en avaient mis à les faire; il n'en demeura pas la plus légère trace. Les lubricités se reprirent ${ }^{12}$ »

9 La restauration du corps et la guérison totale des blessures deviennent ainsi les instruments symboliques de la persécution: comme le personnage lui-même, que son invraisemblable naïveté empêche de tirer un quelconque enseignement des expériences qu'elle traverse, son corps garde la même fraîcheur incroyable, et nécessaire à la répétition programmée des supplices. L'intégrité physique joue donc dans le récit le même rôle que l'ingénuité de Justine: permettre à l'acharnement de l'intrigue de poursuivre son déroulement, et au narrateur-bourreau de continuer à persécuter sa victime, comme il le résume ironiquement à la fin de l'épisode de Rodin ${ }^{13}$ dans La Nouvelle Justine :

«À cela près de cette marque flétrissante..., de quelques vestiges de verges qui, grâce à la pureté de son sang, disparurent bientôt..., de quelques attaques sodomites qui, dirigées par des membres ordinaires, ne la déformaient nullement; à tout cela près, disons-nous, notre héroïne, âgée de dix-huit ans lorsqu'elle sortit de chez Rodin, y ayant d'ailleurs été bien soignée, bien nourrie, n'avait encore rien perdu, ni de ses forces, ni de sa fraîcheur; elle entrait dans cet âge heureux où il semble que la nature fasse un dernier effort pour embellir celle que sa main destine aux plaisirs des hommes ${ }^{14}$."

10 Le goût pour la souffrance et la transformation des libertins en bourreaux caractérise donc aussi la structure énonciative du texte, le narrateur devenant un persécuteur qui s'acharne sur son personnage en offrant tous les moyens d'une attaque systématique 
contre lui. Le droit de supplicier gagne ainsi d'autres sphères que celle de la justice officielle de l'Ancien Régime, largement sous-représentée dans le texte.

Mais ce retournement des valeurs va encore plus loin chez Sade: non seulement les libertins, dans l'exercice de leur plaisir égoïste et cruel, sont assimilés à des bourreaux, mais ils en possèdent également les prérogatives. Omnipotents dans leurs désirs, ils se dotent en effet de moyens - tribunaux, machines de torture, droit de condamner à mort leurs victimes sans procès - qui les transforment en bourreaux officiels, aussi puissants que les exécuteurs publics de l'Ancien Régime. Justine ou les Malheurs de la vertu donne ainsi l'impression de filer jusqu'au bout ce qui n'était au départ qu'une métaphore: le libertin était un « bourreau » parce qu'il aimait faire souffrir, il le devient au plein sens du terme lorsqu'il se dote d'instruments de coercition, et d'une autorité que rien ne semble pouvoir remettre en question. Cette force et ce droit d'infliger les mêmes supplices que ceux qui ont cours dans la justice officielle caractérisent deux personnages du roman : le chirurgien Rodin, rencontré sur la route de l'Isère, et l'évêque de Grenoble.

Rodin apparaît en effet comme un véritable bourreau dans la mesure où il s'est lui-même arrogé le droit d'infliger des châtiments aux enfants qu'il reçoit dans le cadre de son " école ». Cette structure, qui lui offre surtout l'avantage de se constituer une réserve de victimes sexuelles, le transforme en « instituteur pervers ", libre de laisser s'exprimer les élans de sa lubricité. Les scènes de sanction s'assimilent alors, par leur violence et les relations qu'elles instaurent entre le maître et ses " élèves ", à des exécutions, ce que le texte explicite en utilisant le terme de «bourreau » :

«Et pendant ce temps, Rodin très ému, saisit les mains de la jeune fille, il les attache en l'air à l'anneau d'un pilier placé au milieu de la chambre de correction. Julie n'a plus de défense... plus d'autre... que sa belle tête languissamment tournée vers son bourreau, de superbes cheveux en désordre, et des pleurs inondant le plus beau visage du monde ${ }^{15}$.»

13 Le rituel de cette scène, comme le nom donné à la pièce, La Chambre de correction, achèvent de faire du chirurgien un avatar du bourreau officiel, ce qui se confirme lorsqu'il décide, avec son complice, de juger puis de condamner Justine qui les a trahis en tentant de s'enfuir. La jeune femme comparait en effet devant ses juges qui sont aussi ses exécuteurs, si bien que Sade fond ici dans une seule et même scène les deux versants du bourreau, le versant sexuel et celui de la justice :

« «Montons, montons, il faut juger cette affaire-là.» [...] Toute la rage de ces furieux se tourne contre moi, je suis accablée des plus dures invectives, et les plus effrayants arrêts se prononcent; il ne s'agit de rien moins que de me disséquer toute vive ${ }^{16}$."

14 Cette contagion des valeurs s'achève lorsque Rodin décide, au lieu de tuer Justine, de lui infliger une marque au fer rouge, destinée à la faire accuser de vol dès qu'elle aura affaire à la justice. Le libertin s'empare alors d'une prérogative qui appartenait exclusivement aux bourreaux officiels de l'Ancien Régime, qui avait instauré toute une hiérarchie de marques infamantes, en fonction de la gravité de la faute commise :

«Oui, continue-t-il, punissons-la mille fois davantage que si nous prenions sa vie, marquons-la, flétrissons-la; cet avilissement joint à toutes les mauvaises affaires qu'elle a sur le corps, la fera perdre ou mourir de faim; elle souffrira du moins jusque-là, et notre vengeance plus prolongée en deviendra plus délicieuse ${ }^{17}$ ».

15 Plus encore, il joint à la souffrance du châtiment officiel la jouissance proprement sadique puisque la marque au fer permet de prolonger le supplice à l'infini, le corps étant marqué à vie : le libertin utilise donc les instruments et les pouvoirs du bourreau traditionnel au 
service de ses seuls fantasmes, et de cette représentation du plaisir qui fait de lui un "bourreau» au sens cette fois sadien du terme. Cette figure se trouve donc bien à la croisée des deux domaines, ce qui s'observe de manière encore plus frappante au moment de la rencontre de l'évêque de Grenoble.

Alors que Justine retombe entre les mains de la Dubois, qui la fait accuser d'incendie et d'infanticide, cette dernière lui propose de rencontrer l'évêque afin de bénéficier d'un soutien dans le procès qui lui est intenté. Le roman, qui touche presque à sa fin, confère alors à l'ambivalence de la notion de bourreau une importance particulière. Les goûts cruels de l'évêque lui valent immédiatement d'être assimilé à un bourreau par la narratrice: "Les plaisirs de ce monstre était ceux d'un bourreau. Ses uniques voluptés consistaient à trancher des têtes ${ }^{18}$. » Une nouvelle fois, la souffrance infligée aux victimes ne relève pas du hasard mais s'inscrit dans le cadre de la justice officielle, la décapitation étant en effet l'une des condamnations qui étaient régulièrement prononcées à la fin de l'Ancien Régime, en particulier pour les victimes issues des milieux les plus aisés (il s'agit du châtiment traditionnel des nobles). Les goûts lubriques et cruels de l'évêque en font donc un bourreau aux deux sens du terme, ce qu'explicite la troisième version du texte, La Nouvelle Justine, qui approfondit avec plus de détails les épisodes du texte initial. Le lecteur y découvre en effet la guillotine infernale inventée par le personnage, qui se délecte au spectacle des têtes tranchées avec la plus grande lenteur au milieu de son boudoir :

«Du milieu s'élevait un petit échafaud où se trouvait placée une machine assez singulière pour mériter une description. Derrière la machine était un fauteuil placé sur l'échafaud, et destiné au personnage qui voulait faire jouer le ressort de l'infernale manivelle, dont voici le détail. [...] Près de sa main droite était un cordon de soie, qu'il pouvait mouvoir à sa guise : l'agitait-il avec violence; le spectre tenant le sabre coupait net et fort vite la tête offerte à ses coups : tirait-il le cordon doucement ; le sabre tailladait, et ne déchirait plus qu'avec lenteur les ligaments du $\mathrm{cou}^{19}$ ”

17 La description technique très précise achève donc de transformer cette scène de jouissance perverse en parodie d'exécution publique, comme si Sade assimilait définitivement le libertin à un bourreau. L'évêque se projette même dans un avenir politique, lorsqu'il souligne la nécessité de maintenir le peuple dans la crainte avec des supplices aussi nombreux qu'effrayants :

«Vous croyez, mes amis, que les supplices que j'érigerais, dans le cas où je me retrouverais revêtu de quelque autorité, deviendraient infiniment plus rigoureux que ceux qui sont maintenant en usage : assurément, ces supplices seraient, et plus affreux, et plus multipliés, sans doute. Souvenez-vous bien que la soumission du peuple, cette soumission si nécessaire au souverain qui le régit, n'est jamais due qu'à la violence et qu'à l'étendue des supplices ${ }^{20}$. »

18 La dimension «sadique» du plaisir débouche donc sur une définition de ce qui s'apparente au despotisme, si bien que la figure métaphorique du bourreau maintient un lien constant, dans l'œuvre de Sade, entre le fantasme privé et la direction collective d'un pays: seul le degré de publicité de la souffrance recherchée différencie ainsi le libertin cruel du tyran politique.

La Philosophie dans le boudoir

19 Les bourreaux changent en effet de camp dans La Philosophie dans le boudoir : lorsqu'il compose ce texte publié sans nom d'auteur en 1795, Sade décide d'y introduire un pamphlet prétendument au service du nouveau régime révolutionnaire, «Français, 
encore un effort si vous voulez être républicains ». Loin de défendre les idéaux de liberté et d'égalité qui s'imposent désormais dans le discours officiel, il énumère les principes d'un gouvernement despotique, dont la survie implique une transformation systématique des citoyens et de leurs dirigeants en bourreaux.

Cette apologie du despotisme est préparée dans le texte par la situation et les relations des personnages imaginés par Sade : les libertins convoqués dans le boudoir de Mme de Saint-Ange se réunissent en effet pour initier la jeune Eugénie, lui donner une leçon complète de scélératesse, mais cette école de perversion prend rapidement des airs de tribunal compte tenu de la dureté du principal initiateur, Dolmancé. Celui-ci commence en effet par menacer Eugénie lorsqu'il constate, dans les premières pages du texte, la survie chez elle de quelques réticences morales :

« Allons, écoutez-moi, jolie petite élève, ou craignez que, si vous n'êtes pas docile, je n'use sur vous des droits que me donne amplement le titre de votre instituteur ${ }^{21}$.»

21 L'initiation de la jeune femme repose ainsi sur un principe de coercition, même momentané puisque Eugénie adopte rapidement la règle de ses «instituteurs immoraux »; le despotisme couve donc dès le début du texte sous les intentions pédagogiques des séducteurs, et il se confirme lorsque ceux-ci décident de laisser libre cours à leurs désirs sexuels avec Eugénie, quelles que soient les souffrances qu'elle risque d'endurer.

L'éducation libertine s'apparente alors de plus en plus à une exécution, les personnages se réjouissant à l'idée de la douleur de la jeune femme, qui n'hésite pas à qualifier le Chevalier de «bourreau » lorsqu'il la déflore brutalement : «Va, tigre... va, déchire-moi, si tu veux maintenant, je m'en moque, baise-moi, bourreau, baise-moi, je t'adore... ${ }^{22}$ " Même à l'égard d'une femme qui partage leurs valeurs, leurs principes, les libertins ne font donc preuve d'aucune pitié, comparant eux-mêmes leur assemblée à un tribunal rendant des arrêts incontestables, comme le précise Mme de Saint-Ange à Eugénie :

«Baise-moi, je te plains... mais la sentence est prononcée ; elle est sans appel, mon cœur, il faut que tu la subisses ${ }^{23}$ ». La force et la brutalité de leur désir les transforment donc en bourreaux, selon la loi matérialiste des énergies et des forces en présence que nous avons déjà rapidement évoquée, et que théorise encore une fois Dolmancé :

«Il n'y a aucune comparaison entre ce qu'éprouvent les autres et ce que nous ressentons : la plus forte dose de douleur chez les autres doit assurément être nulle pour nous, et le plus léger chatouillement de plaisir, éprouvé par nous, nous touche; donc nous devons à tel prix que ce soit, préférer ce léger chatouillement qui nous délecte, à cette somme immense des malheurs d'autrui, qui ne saurait nous atteindre ${ }^{24}$.»

24 Il est donc logique qu'Eugénie elle-même, comme elle incite le Chevalier à la déflorer en acceptant une douleur pour elle nécessaire à l'obtention d'un plaisir plus grand, se transforme ensuite en bourreau en réclamant une exécution et le sacrifice d'une victime :

MME DE SAINT-ANGE - Quel caprice t'agite, Eugénie ? Dis-le nous avec confiance.

EUGÉNIE, égarée - Je voudrais une victime. [...]

MME DE SAINT-ANGE - Et que lui ferais-tu?

EUGÉNIE - Tout... tout... tout ce qui pourrait la rendre la plus malheureuse des

créatures : oh ! ma bonne, ma bonne, aie pitié de moi, je n'en puis plus ${ }^{25}$.

Avant même la torture de sa propre mère, la jeune femme a donc consenti avec frénésie à la cruauté des bourreaux qui l'entourent: le roman se présente comme une initiation libertine, mais s'achève sur la transformation d'une victime en exécutrice, qui plus est 
raffinée et perverse puisqu'elle exercera sur sa propre famille les lois tyranniques que viennent de lui inculquer ses maitres.

Lorsque Mme de Mistival arrive dans le boudoir pour sauver sa fille de l'école de perversion qui s'est organisée chez Mme de Saint-Ange, il est trop tard : les bourreauxlibertins ont achevé leur œuvre, et la mère tombe dans le piège d'une exécution fomentée par son propre mari, comme le lui signifie la maîtresse des lieux : «Écoute, putain, je vais à la fin t'instruire... Tu es pour nous une victime envoyée par ton mari même, il faut que tu subisses ton sort, rien ne saurait t'en garantir ${ }^{26}$. » Le boudoir devient alors un espace judiciaire, mais qui fonctionne de manière despotique puisque les arrêts qui s'y rendent ne suivent d'autre loi que celle de la fantaisie et de la cruauté des bourreaux :

«Peut-être seras-tu pendue, rouée, écartelée, tenaillée, brûlée vive, le choix de ton supplice dépend de ta fille, c'est elle qui prononcera ton arrêt, mais tu souffriras, catin, oh, oui, tu ne seras immolée qu'après avoir subi une infinité de tourments préalables; quant à tes cris, je t'en préviens, ils seraient inutiles; on égorgerait un bœuf dans ce cabinet, que ses beuglements ne seraient pas entendus ${ }^{27}$. "

Même Augustin, le valet niais et maladroit initialement exclu du boudoir par Dolmancé et ses partenaires, participe à la sentence finale de la mère d'Eugénie. La conversion des libertins en bourreaux est donc achevée, puisqu'elle gagne même les personnages les plus vulnérables et les plus éloignés de la sphère de la cruauté. Sade fait ainsi se succéder différents arrêts contre la victime :

MME DE SAINT-ANGE - Je la condamne à être pendue.

LE CHEVALIER - Coupée, comme chez les Chinois, en vingt-quatre mille morceaux.

AUGUSTIN - Tenez, moi je la tiens quitte pour être rompue vive.

EUGÉNIE - Ma petite maman sera lardée avec des mèches de soufre, auxquelles je me chargerai de mettre le feu en détail.

DOLMANCÉ - Eh bien, mes amis, en ma qualité de votre instituteur, moi j'adoucis l'arrêt ; mais la différence qui va se trouver entre mon prononcé et le vôtre, c'est que vos sentences n'étaient que les effets d'une mystification mordante, au lieu que la mienne va s'exécuter ${ }^{28}$.

Finalement condamnée à être contaminée par la vérole puis infibulée, la mère d'Eugénie se voit renvoyée chez elle sans ménagement par ses bourreaux : le boudoir, à la fin du roman, s'est bien transformé en espace tyrannique, à l'image des principes développés dans le pamphlet qui annonçait cette chute cruelle.

« Français, encore un effort si vous voulez être républicains » associe en effet la figure du citoyen à celle du bourreau. Développant dans une forme de syllogisme les lois qui déterminent selon Sade la naissance d'une république, le texte fait de la cruauté une nécessité dans un système forcément vicié, puisque la liberté ne naîtra pas ex nihilo, mais sur les cendres d'un régime belliqueux et corrompu. La liberté originelle est donc perdue à jamais, si bien que la république qui succède à la monarchie présentée comme tyrannique comportera nécessairement une dimension de violence et d'injustice :

«Une nation qui commence à se gouverner en république, ne se soutiendra que par des vertus, parce que, pour arriver au plus, il faut toujours débuter par le moins; mais une nation déjà vieille et corrompue, qui courageusement secouera le joug de son gouvernement monarchique pour en adopter un républicain, ne se maintiendra que par beaucoup de crimes ; car elle est déjà dans le crime ; et si elle voulait passer du crime à la vertu, c'est-à-dire d'un état violent dans un état doux, elle tomberait dans une inertie dont sa ruine certaine serait bientôt le résultat ${ }^{29}$. »

Le pouvoir de ce nouveau régime aura donc nécessairement une part de bourreau en lui, d'autant que Dolmancé précise que la guerre et le meurtre seront les principaux ressorts 
de cette politique née du mensonge, et qui ne pourra se prolonger que par un autre mensonge. Le citoyen devra alors choisir entre le rôle de victime ou celui de bourreau, dans un système globalement despotique, si l'on se réfère du moins à la définition qu'en donne L'Encyclopédie :

DESPOTISME : f.m. (Droit politique) gouvernement tyrannique, arbitraire et absolu d'un seul homme. [...] Le principe des états despotiques est qu'un seul prince y gouverne selon ses volontés, n'ayant absolument d'autre loi qui le domine, que celle de ses caprices : il résulte de la nature de ce pouvoir qu'il passe tout entier dans les mains de la personne à qui il est confiée ${ }^{30}$.

31 Mais le bourreau n'est pas seulement la figure métaphorique qui résume les principes du despotisme prôné par Sade. Il caractérise aussi la jouissance et la recherche du plaisir en général, si bien que le gouvernement décrit dans le texte ne fait que prolonger les principes égoïstes qui président à la vie intime des citoyens : entre le libertin privé et le tyran officiel, l'élargissement s'établit par la nature même du plaisir, nécessairement despotique puisqu'il exclut toute prise en compte de l'autre :

"Que désire-t-on quand on jouit? Que tout ce qui nous entoure ne s'occupe que de nous, ne pense qu'à nous, ne soigne que nous [...]. Il n'est point d'homme qui ne veuille être despote quand il bande, il semble qu'il a moins de plaisir si les autres semblent en prendre autant que lui [...]. L'idée de voir un autre jouir comme lui le ramène à une sorte d'égalité qui nuit aux attraits indicibles que fait éprouver le despotisme d'alors ${ }^{31}$. »

La double valeur du despotisme et du bourreau, à la fois sexuel et politique, apparaît donc ici d'autant plus clairement que Sade entretient la plus grande confusion entre les différents lexiques employés : les mêmes mots sont en effet utilisés pour caractériser ces deux formes de coercition, intime et publique. La Philosophie dans le boudoir achève donc ce qui n'était qu'esquissé dans Justine, autrement dit la réunion des domaines et la généralisation de la fonction des bourreaux, qui interviennent désormais dans tous les domaines. Sade imagine ainsi une contre-utopie effrayante, celle qui naîtrait du basculement public définitif des libertins, qui mettraient alors la nation tout entière au service de leurs fantasmes de domination et de cruauté. Prenant à rebours la tradition morale de la littérature contemporaine, qui aggrave à l'approche de la Révolution les vices imputables aux libertins et aux aristocrates qui ont provoqué la ruine de la France, il choisit au contraire de renforcer la puissance de ces derniers : se sachant menacés, les libertins sadiens n'ont en effet d'autre choix que de durcir leurs positions, de radicaliser leurs théories, voire d'organiser une résistance clandestine, à l'image de la « Société des amis du crime » décrite dans l'Histoire de Juliette quelques années plus tard. La menace de plus en plus pesante des «bourreaux" de la Terreur amène donc Sade à adopter une réaction de provocation et d'hyperbole: en grossissant à l'extrême les "tares » que le régime impute aux élites de jadis, et en substituant au repli stratégique imposé par la prudence le fantasme de la publicité et de l'exhibitionnisme le plus grand, il rêve d'une France tout entière au service de ses bourreaux, et de ses fantasmes.

Le régime révolutionnaire ne s'y trompera pas, qui condamnera Sade en l'enfermant à Picpus pendant toutes les années de la Terreur. S'il échappe en effet de justesse à la guillotine, l'auteur de Justine apparaît à tous ses contemporains comme un auteur monstrueux, voire un bourreau à part entière. Ses romans sont considérés comme des menaces politiques réelles, comme des incitations à la violence, ce qui pousse certains écrivains, parmi lesquels Rétif de la Bretonne, à chercher à les combattre sur leur propre terrain, autrement dit la représentation de la souffrance. Lorsqu'il publie en 1798 L'Anti- 
Justine ou les Délices de l'amour ${ }^{32}$, Rétif présente son texte comme un antidote au " poison " qu'est Justine ou les Malheurs de la vertu. L'auteur supposé, dénommé Jean-Pierre Linguet, s'en prend de fait nommément à l'œuvre de Sade, réduit à ses initiales Dsds :

«Moi, Jean-Pierre Linguet, maintenant détenu à la Conciergerie, déclare que je n'ai composé cet ouvrage, tout savoureux qu'il est, que dans des vues utiles. L'inceste, par exemple, ne s'y trouve que pour équivaloir, au goût corrompu des libertins, les affreuses cruautés par lesquelles Dsds les stimule [...]. Ce scélérat ne présente les délices de l'amour, pour les hommes, qu'accompagnés de tourments, de la mort même, pour les femmes. Mon but est de faire un livre plus savoureux que les siens, et que les épouses pourront faire lire à leurs maris pour en être mieux servies [...]. Mais l'on en abhorrera davantage le vivodisséqueur... Puisse l'ouvrage que je publie faire tomber les siens ${ }^{33}$ ! »

Il faut donc, dans la lignée générale de l'idéologie révolutionnaire qui se propose de tout régenter, et de rééduquer les citoyens dans l'ensemble des domaines de l'existence privée et publique, réorienter l'imagination des lecteurs : à trop parcourir des scènes de torture et de souffrance, ils risquent de se transformer à leur tour en bourreaux, puisque l'inventeur de tels textes est lui-même nécessairement un persécuteur :

«Ce n'est qu'à force de volupté, de tableaux libidineux, tels que les savoureuses jouissances qui vont suivre, qu'on peut combattre avantageusement, dans le cœur et l'esprit des libertins blasés, les goûts atroces éveillés par les abominables productions de l'infâme et cruel Dsds! Ainsi, je réserve toute ma chaleur pour décrire des jouissances ineffables, au-dessus de tout ce qu'a pu inventer l'imagination exclusivement bourrelle de l'auteur de Justine ${ }^{34}$. "

L'exemple de Sade montre ainsi une rare interaction des domaines : le bourreau est tantôt dans l'œuvre, tantôt en dehors de l'œuvre, mais les autorités révolutionnaires ne peuvent admettre l'idée que l'inventeur de telles contre-utopies libertines ne représente une réelle menace politique pour le régime - on n'a pas une imagination bourrelle sans être, d'une manière indirecte, un ennemi de la Révolution au tournant du XVIII ${ }^{\mathrm{e}}$ siècle.

\section{BIBLIOGRAPHIE}

DIDEROT Denis et al., Encyclopédie ou Dictionnaire raisonné des sciences et des arts, nouvelle impression en fac-similé de la première édition de 1751-1780, Stuttgart-Bad Cannstatt, vol. 4, 1990.

GENAND Stéphanie, Le modèle libertin et la fin de l'Ancien Régime, 1782-1802, Thèse de doctorat de littérature française sous la direction de Michel Delon, université de Paris IV-Sorbonne, 2002, 671 pages.

RESTIF Nicolas, dit Rétif de la Bretonne, L'Anti-Justine ou les Délices de l'amour, dans CEuvres érotiques de Rétif de la Bretonne, « L’Enfer de la BN », Fayard, t. II, 1985.

REY Alain, Dictionnaire historique de la langue française, Le Robert, 1992, réed. 1998.

SADE Donatien Alphonse François, marquis de, Justine ou les Malheurs de la vertu, dans Euvres, éd. Michel Delon, « Bibliothèque de la Pléiade », Gallimard, t. II, 1995. 
SADE Donatien Alphonse François, marquis de, La Philosophie dans le boudoir, dans Euvres, éd. Michel Delon, « Bibliothèque de la Pléiade », Gallimard, t. III, 1998.

\section{NOTES}

* Cette étude fait suite à une thèse de doctorat de littérature française, soutenue le 29 juin 2002 à l'université Paris IV-Paris-Sorbonne sous la direction du Pr Michel Delon, et intitulée Le Modèle libertin et la fin de l'Ancien Régime, 1782-1802, 671 pages.

1. Voir Emmanuel Taïeb, « Portraits du bourreau », Labyrinthe $n^{\circ} 11$, hiver 2002.

2. Le Robert, 1992, rééd. 1998, p. 482.

3. Sade, dans Euvres, éd. Michel Delon, « Bibliothèque de la Pléiade », Gallimard, t. II, 1995.

4. Ibid., t. III, 1998.

5. La Philosophie dans le boudoir, op. cit., p. 67.

6. Justine ou les Malheurs de la vertu, op. cit., p. 379 (c'est nous qui soulignons).

7. Op. cit., p. 380 (c'est nous qui soulignons).

8. Loc. cit. (c'est nous qui soulignons).

9. Qui est de 1787.

10. La Nouvelle Justine, dans CEuvres, op. cit., p. 402.

11. Op. cit., p. 521.

12. Justine ou les Malheurs de la vertu, op. cit., p. 378.

13. Cet épisode est présenté en détail plus loin.

14. La Nouvelle Justine, op. cit., p. 570.

15. Op. cit., p. 204 (c'est nous qui soulignons).

16. Op. cit., p. 221.

17. Op. cit., p. 222.

18. Op. cit., p. 359 (c'est nous qui soulignons).

19. La Nouvelle Justine, dans Euvres, op. cit., p. 1056.

20. Op. cit., p. 1064.

21. La Philosophie dans le boudoir, op. cit., p. 17.

22. Op. cit., p. 108.

23. Ibidem.

24. Op. cit., p. 98.

25. Op. cit., p. 103.

26. Op. cit., p. 169.

27. Ibidem.

28. Op. cit., p. 173.

29. Op. cit., p. 147. Ce passage est une réplique directe au chapitre célèbre de L'Esprit des lois (1748) où Montesquieu définissait la République comme le régime de la vertu.

30. Encyclopédie ou Dictionnaire raisonné des sciences et des arts, nouvelle impression en facsimilé de la première édition de 1751-1780, Stuttgart-Bad Cannstatt, vol. 4, 1990, p. 886.

31. Op. cit., p. 158.

32. Rétif de la Bretonne, dans CEuvres érotiques de Rétif de la Bretonne, "L’Enfer de la BN », Fayard, t. II, 1985.

33. Op. cit., p. 287.

34. Op. cit., p. 303 (c'est nous qui soulignons). 


\section{RÉSUMÉS}

La figure du bourreau est profondément liée à l'œuvre et à la vie du marquis de Sade (1740-1814) ; enfermé la moitié de sa vie dans les prisons de l'Ancien Régime, dans celles de la Révolution puis dans celles du Consulat, il apparaît en effet comme une victime des différents systèmes politiques qui se sont succédé à la fin du XVIIIe siècle. Mais, paradoxalement, c'est pendant ces années de coercition qu'il conçoit ses romans les plus violents, Justine ou les Malheures de la vertu et La Philosophie dans le boudoir. L'intrigue repose toujours sur la persécution de jeunes femmes, aux prises avec de cruels bourreaux qui appuient leur justification de la souffrance sur des théories à la fois physiologiques et politiques : l'autre est nécessairement un objet de soumission, dans un système qui apparaît chez Sade comme très cohérent, puisque la représentation du plaisir rejoint celle de la société et des relations humaines dans leur ensemble. Comment un écrivain condamné à passer sa vie emprisonné a-t-il pu imaginer une vision du monde où les victimes subissent les pires sorts de la part de ceux qu'elles croisent? Comment peut-on, en tant que victime, se fantasmer avec une telle intensité dans un rôle de bourreau?

Executioners

Beteween sexual servitude and political despotism : the role of the bourreau in the works of Sade The figure of the executioner is closely tied to the work and life of the Marquis de Sade (1740-1814): jailed for half of his life in the prisons of the Ancien Regime, of the Revolution, and then of the Consulat, he appears in effect as a victim of the different late eighteenth-century political structures. Yet paradoxically, it was during these years of emprisonment that he conceived of his most violent novels, Justine, or the Misfortunes of Virtue an The Bedroom Philosophers . Intrigue always rests on the persecution of young women oppressed by cruel executioners whose justification for this suffering rests on theories that are at once physiological and political: the other is necesseraly an object of submission in a system that appears very coherent within the works of Sade since the representation of pleasure rejoins those of society and of human relations in their entirety. How could a writer condemmed to spending his life in prison imagine a world where victims submit to the worst imaginable fate on the part of those whom they trust? How can someone, in the role of the victim, fantasize so strongly about the role of the executioner?

\section{AUTEUR}

\section{STÉPHANIE GENAND}

Stéphanie Genand, née en 1975, ancienne élève de l'Ėcole normale supérieure de Fontenay-Saint-Cloud, est agrégée de lettres modernes. Allocataire moniteur normalienne, elle a soutenu le 29 juin 2002 une thèse de doctorat de littérature française, «Le Modèle libertin et la fin de l'Ancien Régime, 1782-1802 », à l'université de Paris IVParis-Sorbonne sous la direction de Michel Delon. Elle sera professeur de lettres en lycée à la rentrée prochaine. Sgenand(at)club-internet.fr 